\title{
ALPES: Um sistema multiagente para análise de produções textuais no contexto de um Debate de Teses
}

\author{
Sabrina Siqueira Panceri ${ }^{1}$, Crediné Silva de Menezes $^{12}$ \\ ${ }^{1}$ Programa de Pós Graduação em Informática - Universidade Federal do Espírito Santo (UFES) \\ Av. Fernando Ferrari, 514, Goiabeiras - Vitória - ES - Brasil - Caixa Postal 29075-910 \\ ${ }^{2}$ Faculdade de Educação - Universidade Federal do Rio Grande do Sul \\ Av. Paulo Gama, s/n, Farroupilha - Porto Alegre - RS - Brasil - Caixa Postal 90046-900 \\ \{sabrinapanceri, credine\}@gmail.com

\begin{abstract}
The interactions made in the implementation of pedagogical architecture Debate Theses are carried through the production of texts. This paper investigates the application of Debate Thesis and identifies a number of interventions that can be performed by the mediator, which are of fundamental importance to support the construction of students' knowledge. In order that these activities are performed with the desired quality, we suggest building tools based on research in the context of Natural Language Processing and Information Retrieval, providing a Multi-Agent System called ALPES.
\end{abstract}

Resumo. As interações ocorridas durante a aplicação da arquitetura pedagógica Debate de Teses são realizadas através da produção de textos. Este trabalho investiga a aplicação do Debate de Teses e identifica mediações pedagógicas que podem ser praticadas pelo mediador, e que se constituem de fundamental importância para apoiar a construção de conhecimento dos estudantes. Para que essas atividades possam ser realizadas com a qualidade desejável, sugerimos a construção de ferramentas baseadas em pesquisas no contexto do Processamento de Linguagem Natural e Recuperação de Informação, constituindo um Sistema Multiagente denominado ALPES.

\section{Introdução}

A construção de conhecimento, segundo a epistemologia genética (Piaget apud [Ramozzi-Chiarottino 1988]), requer que os sujeitos realizem interações que provoquem desequilíbrios. Interações ocorrem a todo instante, com os objetos do mundo real, com outras pessoas, com as nossas construções conceituais e cada uma realiza suas contribuições desde que o sujeito se disponha a buscar respostas para as indagações decorrentes. Na escola, as interações com os colegas tem o seu papel e importância, assim como com o professor, como um parceiro com mais experiência que deve buscar intencionalmente criar condições para que seus alunos aprofundem seus conhecimentos.

Ao criar tais condições, o professor tem a intenção de gerar situações de desequilíbrio, para que o aluno busque as informações necessárias para entrar em equilíbrio novamente, consolidando seu conhecimento sobre determinado assunto. Dentro dessa perspectiva o professor assiste de perto à construção do conhecimento de cada aluno, analisando e orientando as buscas. 
Quando se trata de produções textuais, a quantidade de tempo para análise e produção das respostas (feedback) desejadas pelos alunos é ainda maior, pois além do tempo despendido para ler as produções, o professor precisa de tempo para realizar a interpretação daquele texto e gerar sua análise e resposta para cada texto lido.

Considerando o cenário da educação apoiada pelo uso das tecnologias digitais da informação e comunicação, [Nevado et al. 2011] propõe a arquitetura pedagógica (AP) Debate de Teses $^{1}$ que visa a construção do conhecimento através das interações entre o aluno e seus pares. As interações nessa AP são realizadas através da produção de textos relacionados ao tema, a partir de teses propostas pelo mediador (professor). No decorrer da aplicação dessa AP, o mediador terá várias produções textuais para analisar se estão de acordo com o tema proposto pela tese, se possuem os elementos necessários e característicos de cada etapa, além de compreender as produções para elaborar ofeedback para os participantes.

Este artigo está organizado da seguinte forma: na próxima seção apresenta-se o contexto do problema. Na seção 3 são descritos os trabalhos relacionados a esta proposta. O esboço da solução é apresentado na seção 4 e as considerações finais são apresentadas na seção 5 .

\section{Contexto do Problema}

A arquitetura pedagógica Debate de Teses (DT) destina-se a apoiar a construção do conhecimento individual ou coletivo sobre determinado assunto, mediante as interações realizadas no decorrer da mesma, tendo o suporte computacional como meio para sua aplicação. A intenção é que o participante, partindo de seu conhecimento prévio, estenda e aprofunde esses conhecimentos através de interações com seus pares, seguindo uma determinada dinâmica. Nestas interações, que são realizadas através da produção de textos, os participantes expõem suas convicções sobre determinada(s) tese(s) proposta pelo mediador.

A DT tem quatro perfis de atores: Administrador, Mediador, Argumentador e Revisor. Os papéis de administrador e mediador são atribuídos ao professor. Os papéis de argumentador e revisor são atribuídos aos alunos. Em um instante o aluno prepara a sua argumentação sobre as teses propostas, posteriormente ele trabalha como revisor, sobre as argumentações realizadas por seus pares.

Além disso, a DT é composta por quatro fases, que são: (1) posicionamento e argumentação inicial, (2) revisão de pares, (3) réplica e (4) argumentação final. Durante a fase (1) posicionamento e autoria da argumentação inicial, o argumentador deve se posicionar favorável, parcialmente favorável ou contra a tese proposta, e desenvolver a sua argumentação baseando-se em seu conhecimento prévio e em referências teóricas sobre o assunto. Após a fase (1), o participante assume o papel de Revisor, e terá acesso as argumentações realizadas por dois de seus pares. No momento da revisão (2), o revisor, analisa se a argumentação está devidamente apoiada em evidências e se a argumentação está logicamente bem encadeada, buscando apoiar a aprendizagem do colega. Não se trata, por exemplo, de contrapor a sua visão com a do argumentador.

Após finalizada a etapa de revisão, o participante tem acesso às revisões feitas sob

\footnotetext{
${ }^{1}$ Um protótipo funcional dessa arquitetura está disponível em: www.pead.faced.ufrgs.br/debate
} 
sua argumentação inicial, e inicia-se a fase de réplica (3), onde o mesmo deve responder aos questionamentos ou críticas levantados na etapa de revisão. Finalmente, após realizar a réplica, o participante deve realizar a sua argumentação final sobre a tese (4). Neste momento, ele indica novamente o seu posicionamento e refaz a sua argumentação. Espera-se que ao término dessa interação orientada, o aluno (participante) tenha adquirido novos conhecimentos sobre o tema abordado pela tese.

\subsection{Mediação}

Supondo que a AP Debate de Teses seja utilizada em uma turma com 30 (trinta) alunos, serão realizadas as seguintes quantidades de produções textuais para cada tese proposta: 30 argumentações iniciais, 60 revisões, 60 réplicas e 30 argumentações finais. Ao final das interações, o mediador terá acompanhado a produção de 180 textos, e ainda deverá analisar, compreender e julgar se cada produção está de acordo com o tema proposto pela tese e se foram produzidas de acordo com os objetivos de cada etapa.

Várias intervenções podem ser utilizadas em parceria com essa AP com o objetivo de analisar, compreender e fornecer o feedback para os alunos. Na listagem a seguir apresentamos possíveis atividades a serem realizadas pelo mediador:

- Analisar o padrão de respostas de cada participante: Verificar se os textos produzidos pelo participante estão de acordo com cada etapa do debate, se são diferentes e se possuem os elementos necessários a cada fase.

- Identificar "pontos importantes"para orientar a fase de reflexão: Neste caso, ao analisar as conclusões (posicionamento final) de cada aluno, podem ser identificados pontos que não foram compreendidos ou abordados pelos participantes, e esses serem os pontos principais para condução da fase de reflexão sobre o assunto.

- Verificar a evolução das argumentações: Ao analisar a argumentação inicial e o posicionamento final de cada participante, o mediador verifica quais tópicos sobre o assunto proposto pela tese foram desenvolvidos e/ou evoluídos do decorrer da aplicação da AP.

- Avisar sobre o prazo de envio das revisões e réplicas: O mediador envia lembretes para os participantes que ainda não tiverem realizado as atividades quando faltar um (1) dia para o encerramento da fase.

- Elaborar uma síntese das argumentações finais para realizar o feedback: Ao analisar os posicionamentos finais, o mediador pode elaborar uma resposta única, baseada nas construções realizadas pelos participantes para respondê-los.

- Agrupar participantes por posicionamento inicial: Esse agrupamento servirá de base para ajudar na distribuição dos revisores.

- Agrupar participantes que possuem argumentações iniciais semelhantes: Esse agrupamento servirá para auxiliar o mediador a compreender o nível de conhecimento da turma sobre o assunto abordado pela tese, quais possuem conhecimentos semelhantes, e servirá de apoio para distribuição dos revisores.

- Fazer comentários sobre a argumentação inicial para orientar o participante: $O$ mediador pode realizar comentários sobre as argumentações iniciais dos participantes, visando provocá-los a desenvolver a argumentação com outros elementos, ampliando a sua explicação e mesmo orientando o participante caso ele não tenha construído sua argumentação relacionada ao assunto da tese. 
- Distribuir revisores que tenham argumentações diferentes: Essa intervenção visa a distribuição de revisores que tenham posicionamentos e argumentações diferentes, fazendo com que o participante entre em desequilíbrio e busque mais informações para realizar a revisão de uma argumentação que é contrária a sua opinião e que não possua elementos semelhantes a sua argumentação.

- Verificar se as revisões feitas pelo participante são diferentes e relevantes: O mediador analisa as revisões feitas por um mesmo participante buscando se ele questiona a argumentação e faz críticas. Além de verificar se as duas revisões são diferentes e relacionadas a argumentação a que está vinculada.

- Conferir se as réplicas respondem aos questionamentos feitos nas revisões: O mediador analise as réplicas feitas pelos participantes buscando respostas aos questionamentos e críticas apontadas nas revisões.

- Agrupar participantes que possuem argumentações finais semelhantes: Esse agrupamento é realizado a fim de identificar quais participantes chegaram a conclusões semelhantes sobre o assunto abordado pela tese.

- Verificar qual o grau de influência das revisões e réplicas na argumentação final: Essa análise é feita para verificar quais elementos foram inseridos na argumentação final que foram apresentados nas fases de revisão e réplica, assim pode-se verificar qual o nível de influência as trocas realizadas nessas etapas para a construção da argumentação final do participante.

- Identificar quais teses podem ser trabalhadas a partir das argumentações finais: Através da análise dos agrupamentos realizados com as argumentações finais, o mediador identifica quais foram os assuntos que ficaram mais e menos evidentes nas produções, e escolhe quais as próximas teses que podem ser trabalhadas sobre este tema para auxiliar na construção do conhecimento dos participantes.

- Verificar se houve plágio nas revisões, réplicas e argumentações finais: Verifica se os textos produzidos nas fases de réplica, revisão e argumentação final, buscando textos produzidos por outros participantes a fim de evitar cópias e montagens, e incentivar as criações individuais de cada participante.

- Verificar se houve cordialidade na redação das revisões e réplicas: Previne e alerta os participantes caso sejam identificados termos de baixo calão (palavrões), ou comparações entre as argumentações revisadas que as denigram.

Realizar essas mediações de forma manual, analisando e compreendendo cada texto e fazendo as anotações necessárias para realizar cada intervenção consomem um tempo relevante do professor e tornam a realização dessas intervenções impraticáveis, tendo em vista que o professor desenvolve outras atividades laborais além do acompanhamento dessa atividade em específico. Uma forma de diminuir o tempo gasto na execução dessas atividades e tornar viável a prática dessas intervenções é utilizar a tecnologia para apoiar na realização dessas tarefas. Neste contexto, propõe-se a construção de um ambiente para análise das produções textuais, que auxiliará o professor na realização dessas atividades e na produção do feedback esperado pelos participantes (alunos).

\section{Trabalhos relacionados}

Trabalhos relacionados diretamente com a proposta deste artigo, não foram encontrados durante o processo de pesquisa inicial para sua elaboração. Contudo, alguns trabalhos podem ser associados a este tema, por utilizarem os textos produzidos em Ambientes 
Virtuais de Ensino e Aprendizagem (AVEA) para retirada de conhecimento necessário para responder a outras questões, como a expressão de dúvidas, ou mesmo a presença social dos alunos.

[Rigo et al. 2013] fala sobre a identificação de expressões de dúvidas através das análises realizadas nas produções textuais. São utilizados recursos semânticos adquiridos com a utilização da representação de conhecimentos com ontologias e recursos linguísticos utilizando-se de técnicas de processamento de linguagem natural.

[Silva et al. 2012] mostra como a análise dos textos produzidos em AVEA dá suporte ao reconhecimento da presença social do aluno naquele ambiente, identificando assim se o mesmo está interagindo e de dedicando a construção de seu aprendizado.

[Novais and Paraboni 2013] apresenta a combinação de técnicas de processamento de linguagem natural (PLN) com regras de análise estatística para obter um melhor resultado, tendo em vista que o idioma Português possui muitas palavras e ambiguidades, o que dificulta as criações automáticas, além de ser o primeiro trabalho a utilizar a técnica de Factored language models - FLM-based para língua portuguesa.

[Sterbini and Temperini 2013] apresenta o ambiente web, OpenAnswer, capaz de auxiliar o aluno durante o desenvolvimento de respostas discursivas. Neste caso, o ambiente pode ser entendido como genérico, pois, ao inserir as perguntas o professor deve inserir também padrões de respostas que serão utilizados posteriormente como base para realizar as correções das respostas enviadas pelos alunos. A análise dos textos (respostas) leva em consideração à similaridade entre texto inserido pelo aluno, o texto de resposta padrão inserido pelo professor, e a similaridade com as respostas dos outros alunos.

[Iandoli et al. 2014] apresentam uma ferramenta denominada Debate Dashboard. Essa ferramenta é integrada ao sistema Cohere. No artigo são apresentados argumentos e evidências empíricas que mostram que as ferramentas para ambientes colaborativos com suporte computacional para visualização de argumentações podem ser desenvolvidas para apresentar mapas conceituais sobre as interações realizadas pelo participante com o objetivo de fornecer a cada um a possibilidade de acompanhar o seu desenvolvimento dentro do debate, quais foram suas contribuições, quais relações foram realizadas com os argumentos feitos por ele. Importante ressaltar, que o Debate Dashboard auxilia na construção de deliberações coletivas, ou seja, fornece suporte para retirada de afirmações relevantes para o tema em debate que sejam suficientes para criar um entendimento coletivo do que foi discutido.

\section{Esboço da Solução}

A solução proposta por este artigo é apresentada na figura 1. A arquitetura proposta segue o modelo Cliente x Servidor, onde temos na camada cliente o acesso ao sistema Debate de Teses e o acesso as soluções produzidas pela camada servidor. Na camada servidor ficam armazenados os dados do sistema, e o Sistema Multiagente (SMA).

O SMA foi projetado com o objetivo de auxiliar em vários processos que são realizados pelos professores ao analisarem as interações ocorridas durante o DT. Os agentes que compõem o SMA são autônomos e trabalham sobre os dados inseridos pelos alunos durante a realização do DT. Segue a explicação detalhada das funcionalidades de cada agente. 


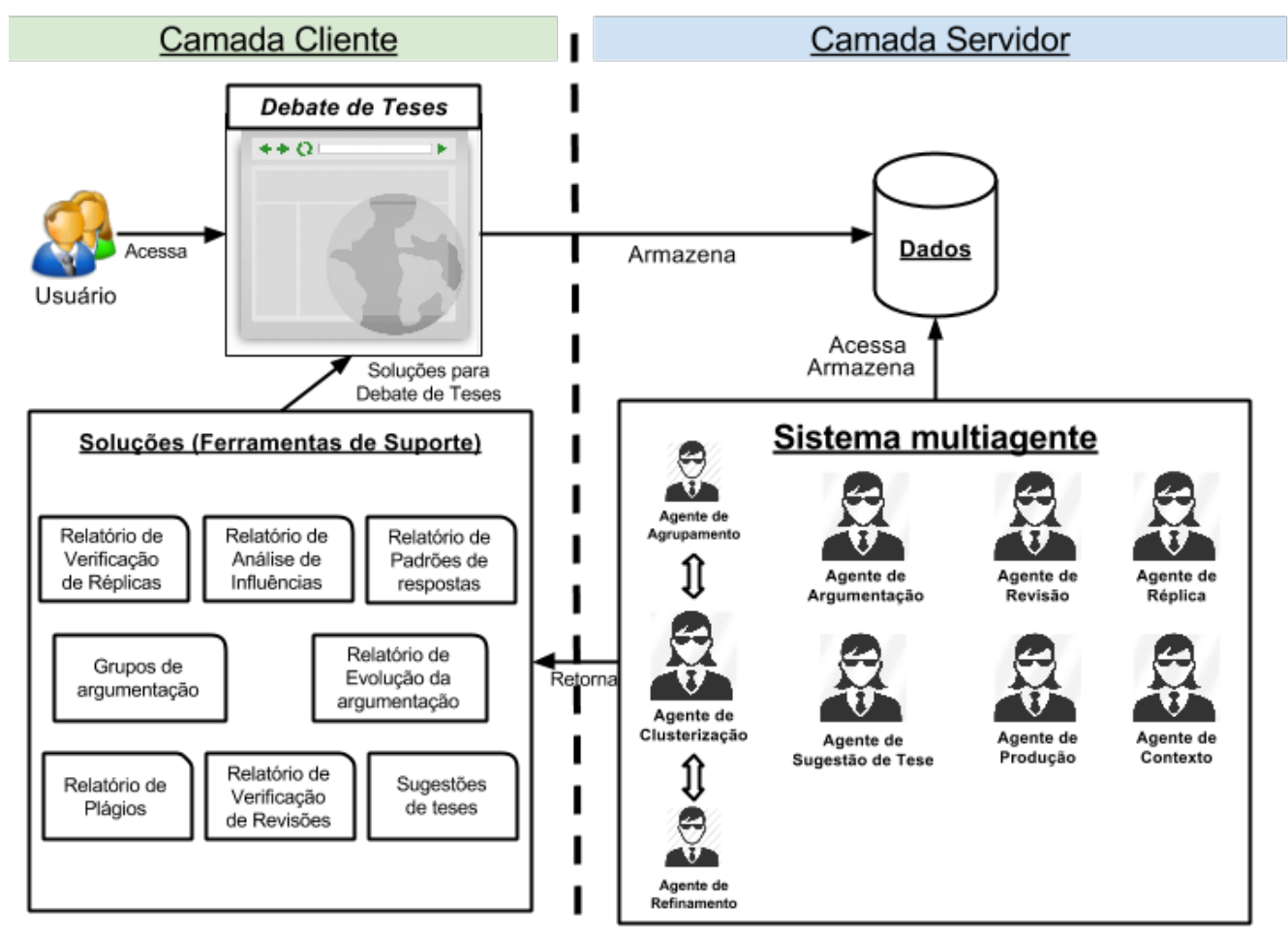

Figura 1. Arquitetura geral da proposta

- Agente de Clusterização: Controla as tarefas realizadas pelos agentes de agrupamento e refinamento. E por iniciar a execução dos mesmos no sistema.

- Agente de Agrupamento: Realiza a comparação dos textos de qualquer fase do debate, agrupando-os em grupos semelhantes. As comparações são realizadas par a par, e os agrupamentos ocorrem quando os textos possuem um grau de similaridade maior ou igual a $70 \%$.

- Agente de refinamento: Efetua o refinamento dos textos agrupados, verificando se não houveram agrupamentos indevidos, e se os textos que pertencem a um mesmo grupo possuem o grau de similaridade correto.

- Agente de Argumentação: Verifica se a argumentação, inicial ou final, está de acordo com o posicionamento indicado pelo usuário. Conferi se há coerência e consistência no texto e se está de acordo com o tema da tese.

- Agente de Revisão: Verifica se a revisão realizada está de acordo com a argumentação em revisão, se a revisão é relevante ${ }^{2}$, se está dentro do tema em discussão e verifica se há cordialidade na escrita. Verifica também se as revisões feitas por determinado aluno são diferentes.

- Agente de Réplica: Monitora se os questionamentos / indicações realizadas nas revisões estão sendo respondidos. Verifica também se o texto de réplica sofreu muitas alterações diante das revisões, ou seja, se houve influência das revisões nesta produção.

\footnotetext{
${ }^{2}$ Neste caso verificamos por exemplo se o texto não é apenas um "concordo com sua opinião"ou "Acho que você está certo".
} 
- Agente de Produção: Monitora as produções realizadas verificando se a sua réplica ou argumentação final não é um recorte das autorias realizadas por outros alunos que foram revisados por este aluno.

- Agente de Contexto: Verifica se houveram modificações relevantes na semântica do texto produzido na argumentação inicial comparado com o texto produzido na argumentação final.

- Agente de Evolução: Verifica a evolução das autorias produzidas pelo aluno dentro do contexto do tema abordado pela tese. Neste caso, é verificado o nível de alterações entre argumentação inicial e final de um aluno.

- Agente de Sugestão de Tese: Realiza indicações de novas teses para o professor, a partir dos agrupamentos realizados tendo como base a análise das argumentações finais. Ele verifica qual é o tema central dos agrupamentos e indica estes temas como possíveis teses a serem tratadas sobre o assunto.

As soluções geradas pelos agentes são apresentadas na forma de relatórios para o usuário. A princípio foram identificadas oito ferramentas de suporte ao trabalho do professor mediador. São elas:

- Relatório de Verificação de Réplicas: Mostra ao professor mediador se as réplicas realizadas responderam de fato as revisões feitas à argumentação inicial. Com isso, o professor poderá identificar quais são os participantes que estão de fato realizando a réplica e respondendo as críticas indicadas nas revisões.

- Relatório de Análise de Influências: Apresenta o nível de influência das revisões sobre as réplicas, ou seja, como as réplicas se diferem da argumentação inicial tendo como base os pontos destacados pelas revisões. Esse relatório pode ajudar o professor a acompanhar o desenvolvimento do debate, pois com as indicações realizadas pela ferramenta ele pode realizar as intervenções necessárias àqueles participantes que não estiverem realizando a etapa da maneira correta.

- Relatório de Evolução da Argumentação: Exibe como houveram alterações entre a argumentação inicial e a argumentação final. Ela considera as interações que o participante teve com outros contextos ao revisar seus pares e construir suas réplicas, comparando o posicionamento e argumentação final, com os elementos de interação, e como eles estão presentes na argumentação final. Essa ferramenta é importante, por gerar indicadores da evolução do aluno, ajudando o professor na tarefa de análise das construções de conhecimento realizadas pelos participantes.

- Grupos de argumentação: Revela o resultado do monitoramento realizado pelo agente de clusterização, tendo como base os textos produzidos nas argumentações finais ou iniciais. Essa solução é importante, pois ao ter acesso a agrupamentos das argumentações enviadas pelos alunos, o professor mediador poderá traçar estratégias de suporte para auxiliar na aprendizagem daquele grupo, ou mesmo identificar que determinado grupo não realizou as argumentações dentro do tema proposto pela tese.

- Relatório de Padrões de respostas: Informa ao professor quais participantes realizaram as mesmas revisões em pares diferentes, facilitando assim o monitoramento das contribuições efetivas.

- Relatório de Plágios: Indica se os textos produzidos por um determinado participante é derivado de outros textos a que aquele participante teve acesso na fase de revisão. Esse monitoramento pode ser realizado sobre os textos produzidos na fase de réplica e argumentação final. 
- Relatório de Verificação das Revisões: Faz a análise das revisões buscando elementos de questionamento e/ou crítica à argumentação em revisão. Além de verificar se a revisão realizada não é apenas uma frase de concordância, como por exemplo "Concordo com sua opinião", e se foi escrita de forma cordial.

- Sugestões de teses: Expõe para o professor mediador indicações de novas teses, correlatas a em análise, e que poderão ser utilizadas para ajudar na construção do conhecimento do alunos. Essas sugestões de teses serão retiradas dos textos produzidos nas argumentações finais, após serem feitos os agrupamentos por similaridade.

A arquitetura proposta indica o desenvolvimento de várias ferramentas de suporte, e dentre elas, este artigo apresenta a descrição detalhada da solução Grupos de Argumentação, que é gerada a partir do funcionamento do Agente de Clusterização e seus agentes auxiliares, agente de agrupamento e agente de refinamento.

Como dito anteriormente, o agente de clusterização é responsável controlar o funcionamento dos agentes de agrupamento e refinamento. A figura 2, apresenta o schema de funcionamento deste agente.

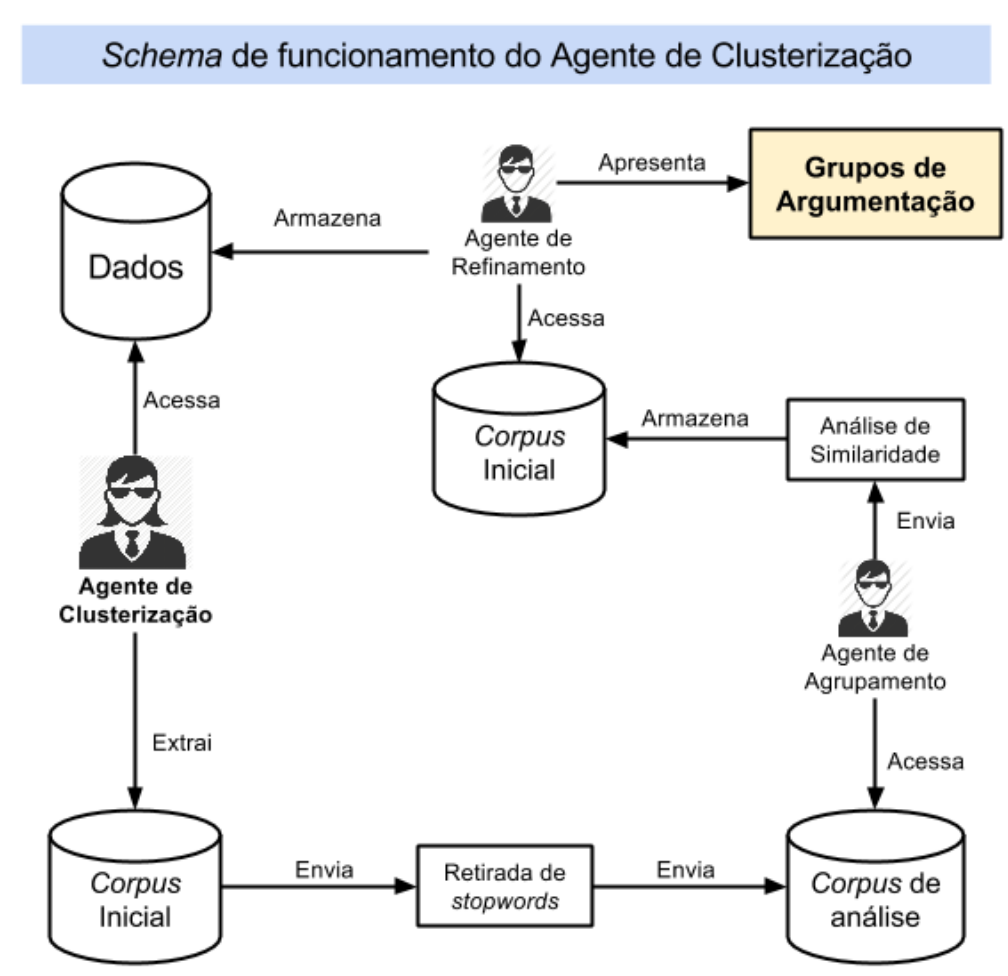

Figura 2. Schema de funcionamento do Agente de Clusterização

O agente de clusterização acessa os dados indicados pelo professor mediador. Neste caso, os dados que podem ser agrupados são a argumentação inicial ou final. Na análise das argumentações iniciais, o professor mediador pode identificar quais alunos possuem conhecimento prévio semelhante sobre o assunto abordado. Com isso o professor pode fazer a indicação dos revisores que pertencerem a um outro grupo, criando uma situação de desequilíbrio para os revisores, que terão que buscar mais informações para elaborar as revisões. Na análise das argumentações finais, a solução mostra ao pro- 
fessor mediador quais alunos chegaram a conclusões semelhantes, auxiliando o professor mediador na produção do feedback para os alunos.

Após realizar acesso aos dados, o agente de clusterização extrai o corpus inicial, que é composto pelos textos das argumentações inicial ou final dos alunos. Em seguida envia o corpus inicial para o processo de retirada das stopwords. Esse processo é composto por algoritmos que realizam a exclusão de termos classificados como artigos, pronomes, proposição, conjunção, interjeição, etc. A classificação dos termos será feita com a integração do parse disponibilizado pelo projeto VISL $^{3}$. Ao final desse processo temos o corpus de análise. Neste momento, o agente de clusterização inicia o processo do agente de agrupamento que fará o acesso ao corpus de análise e dará início a análise de similaridade, que é realizada através da comparação entre todos os textos produzidos e a tese ao qual estão vinculados. Será atribuído ao corpus de análise "A" o grau de similaridade dele para com os demais corpus analisados. Para atender a qualquer contexto, os algoritmos dão suporte a análise léxica dos corpus. Ao finalizar as comparações, são armazenados os valores de similaridade entre os textos, e a partir disso tem-se o agrupamento inicial. Nesta etapa, o agente de clusterização inicia o processo do agente de refinamento que terá como tarefa conferir se os textos agrupados em um mesmo grupo possuem o nível de similaridade desejável. Ao final da conferência, ele deve armazenar os Grupos de Argumentação gerados no banco de dados, e enviar para a camada do servidor o relatório para ser apresentado ao usuário.

\section{Considerações Finais}

Auxiliar no processo de análise e correção dos textos produzidos nas interações em ambientes virtuais de ensino e aprendizagem é de grande importância para apoiar os professores no acompanhamento da evolução do aluno ou da turma, na produção dos feedbacks, e principalmente na economia do tempo gasto para análise e correção das atividades propostas.

No desenvolver da aplicação da arquitetura pedagógica Debate de Teses, a produção dos textos produzidos nas fases de argumentação inicial e final indicam como está o conhecimento do aluno naqueles momentos, e o suporte de ferramentas capazes de agrupar os alunos pelo nível de semelhança de seus conhecimentos prévios ou finais é relevante quando considera-se que com esse tipo de informação o professor pode traçar estratégias individuais para auxiliar os grupos de alunos na construção de seu conhecimento, o que geralmente não é abordado devido a quantidade de alunos e a falta de tempo dos professores para utilizar estratégias de acompanhamento individuais.

O arcabouço de ferramentas disponibilizadas para apoiar as intervenções do mediador durante a aplicação da AP Debate de Teses evidenciam possibilidades de utilização da tecnologia para facilitar a execução de atividades que apoiem a construção de conhecimento.

Espera-se que com esse aporte tecnológico o mediador (professor) realize as intervenções: Identificar quais teses podem ser trabalhadas a partir das argumentações finais; Elaborar uma síntese das argumentações finais para realizar o feedback; Identificar "pontos importantes" para orientar a fase de reflexão.

\footnotetext{
${ }^{3}$ Link do projeto: http://beta.visl.sdu.dk/visl/pt/parsing/automatic/
} 


\section{Referências}

Iandoli, L., Quinto, I., De Liddo, A., and Buckingham Shum, S. (2014). Socially augmented argumentation tools: Rationale, design and evaluation of a debate dashboard. Int. J. Hum.-Comput. Stud., 72(3):298-319.

Nevado, R. N., Menezes, C. S. d., and Vieira Jr, R. R. M. (2011). Debate de teses uma arquitetura pedagógica. In Anais do $22^{\circ}$ Simpósio Brasileiro de Informática na Educação, volume 1, pages 820-829.

Novais, E. M. d. and Paraboni, I. (2013). Portuguese text generation using factored language models. Journal of the Brazilian Computer Society, 19(2):135-146.

Ramozzi-Chiarottino, Z. (1988). Psicologia e Epistemologia Genética de Jean Piaget. São Paulo: EPU.

Rigo, S. J., Flores, E. M., Abech, M., Barbosa, J. L. V., Costa, C., Martins, L. L., Oliveira, T. B., and Araujo, D. A. (2013). O papel do processamento de língua natural e da representação de conhecimento na extração de informações em mensagens textuais na educação à distância. In Anais do $24^{\circ}$ Simpósio Brasileiro de Informática na Educação, volume 1, pages 647-656.

Silva, J. K. K. d., Bastos, H. P. P., Bercht, M., and Wives, L. K. (2012). Automatização do processo de identificação de presença social em fóruns e chats. In Anais do $23^{\circ}$ Simpósio Brasileiro de Informática na Educação, volume 1.

Sterbini, A. and Temperini, M. (2013). Openanswer, a framework to support teacher's management of open answers through peer assessment. In Frontiers in Education Conference, 2013 IEEE, pages 164-170. 